\title{
Broad-Spectrum Model for Sharing Analysis between IMT- Advanced Systems and FSS Receiver
}

\author{
Lway Faisal Abdulrazak ${ }^{1}$, Kusay F. Al-Tabatabaie ${ }^{2}$ \\ ${ }^{1 \& 2}$ Lecturer at the Computer Science Dep., Cihan University -Sulaimanyia Campus, Iraq
}

\begin{abstract}
An appraisal of orthogonal frequency division multiplexing (OFDM) accredited for IMT-Advanced has been well thought-out in this letter. Derivation of the power spectral density (PSD) produce new model which easily assess the interfering signal power that appears in the band of a victim system without a spectrum emission mask. Furthermore, the broad-spectrum investigative model (BIM) can assess the interference from the $4 G$ systems into FSS systems, when transmit power is unallocated to some sub-carriers overlapping the band of the victim system. Closed form is derived to create the model.
\end{abstract}

Keywords: IMT-Advanced; Fixed satellite services; coexistence; OFDM; spectrum.

\section{Introduction}

Three basic schemes dedicated to analysis of the interference potential between systems that have been addressed in previous work [1] [2], namely, the minimum coupling loss (MCL) method, the advanced minimum coupling loss (A-MCL) and the Monte Carlo method. First, the MCL method utilizing diagnostic estimation is simple to use and can be constructed in less time than the other models. The method does not require a computer for implementation. However, this method can be only adopted as a special case of the "static" interference situations [3].

The coexistence study [4] applied a spectrum emission mask, an essential parameter for adjacent frequency sharing analysis, to calculate the attenuation of the interference signal power in the band of the victim system. However, that method can not be utilized in assessing interference caused by $4 \mathrm{G}$ systems for which there is no spectrum emission mask. Moreover, MCL not capable to evaluate the $4 \mathrm{G}$ interference with other systems even if there is a spectrum mask in a case that transmit power is not allocated to some subcarriers overlapping the band of the victim system to mitigate $4 \mathrm{G}$ interference to other systems. The aim of this study is the maturity broad-spectrum investigative model for evaluation the interference from 4G OFDM-based systems. In South Korea they invented advanced minimum coupling loss method (AMCL) but they ignored the complexity of real sinusoidal signal, this paper will tune there vision into a more applicable way through triangular technique [5].

\section{Proposed Method}

According The minimum required loss in $\mathrm{dBW}$ is described by

$\mathrm{Lmin}=\mathrm{Pt}+\mathrm{Gt}+\mathrm{Gr}+\mathrm{Lr}-\mathrm{Imax}$

where $\mathrm{Pt}$ is the transmit power of interferer $(\mathrm{dBW})$ in the reference bandwidth and Imax is the maximum permissible interference power (about $-166 \mathrm{dBW}$ ) in the reference bandwidth to be exceeded for no more than $\rho$ $\%$ of the time at the terminals of the antenna of receiving FSS. The antenna gains are to be Gt and Gr for the interfering transmitter and the victim receiver in $\mathrm{dBi}$, respectively. $\mathrm{Lr}$ is the interfering signal power loss, which accounts for the fraction of interfering signal power that appears in the band of the victim system with $\mathrm{dBw}$ scale. The interfering signal power loss Lr for the interfering system with OFDM is derived through a power spectral density (PSD) analysis of the OFDM signals. Figure 1 shows that the PSD of 4G OFDM-based system overlaps the PSD of FSS.

Assuming an OFDM system having M subcarriers and a rectangular pulse, the PSD of the OFDM signal is represented as

$$
S_{S}(f)=\sum_{i=0}^{M-1} \frac{P_{S}}{R_{S}} \sin c^{2}\left(\frac{f}{R_{S}}-i\right)
$$

Where Ps is the power of a single OFDM subcarrier and Rs is the subcarrier spacing. Here, the PSD of the broadcasting system is triangular.

$$
S_{b}(f)=\frac{P_{b}}{W_{b}} \operatorname{tri}\left(\frac{f}{2 W_{b}}\right)
$$


Where $\mathrm{P}_{\mathrm{b}}$ and $\mathrm{W}_{\mathrm{b}}$ are the transmit power and bandwidth of broadcasting systems, respectively. Figure 1 shows that the PSD of OFDM-based IMT-Advanced system overlaps the PSD of broadcasting satellite systems. The interfering signal power attenuation by the bandwidth overlapping ratio can be expressed by integration of the three areas shown in the above figure as

$$
\begin{aligned}
& L_{a t t}=10 \log _{10}\left(\frac{\int_{f_{c}-W_{b} / 2}^{f_{c}+W_{b} / 2}\left[A_{1}(f)+A_{2}(f)+A_{3}(f)\right] d f}{P_{t}}\right) \\
& L_{a t t}=10 \log _{10}\left(\frac{\int_{f_{c}-W_{b} / 2}^{f_{c}-W_{b} / 4} A_{1}(f) d f+\int_{f_{c}-W_{b} / 4}^{f_{c}+W_{b} / 4} A_{2}(f) d f+\int_{f_{c}+W_{b} / 4}^{f_{c}+W_{b} / 2} A_{3}(f) d f}{P_{t}}\right) \\
& A_{1}(f)=\left(C_{1} f+C_{2}\right) S_{S}(f) A_{2}(f)=S_{S}(f) \\
& A_{3}(f)=\left(-C_{1} f+C_{3}+P_{S}\right) S_{S}(f)
\end{aligned}
$$

$\mathrm{C} 1=4 \mathrm{PS} / \mathrm{Wb}, \mathrm{C} 2=(4 \mathrm{PS} / \mathrm{Wb})(\mathrm{fc}-(\mathrm{Wb} / 2))$ and $\mathrm{C} 3=(4 \mathrm{PS} / \mathrm{Wb})(\mathrm{fC}+(\mathrm{Wb} / 4))$

$$
\begin{aligned}
\int_{f_{c}-W_{b} / 2}^{f_{c}-W_{b} / 4} A_{1}(f) d f= & \int_{f_{c}-W_{b} / 2}^{f_{c}-W_{b} / 4}\left(C_{1} f+C_{2}\right) S_{S}(f) d f \\
& =\int_{f_{c}-W_{b} / 2}^{f_{c}-W_{b} / 4}\left(C_{1} f+C_{2}\right) \sum_{i=0}^{M-1} \frac{P_{S}}{R_{S}} \sin c^{2}\left(\frac{f}{R_{S}}-i\right) d f \\
& =\sum_{i=0}^{M-1} \frac{P_{S}}{R_{S}}\left[\begin{array}{l}
C_{1} \int_{f_{c}-W_{b} / 2}^{f_{c}-W_{b} / 4} f \frac{\sin ^{2}\left[\pi\left(\frac{f}{R_{S}}-i\right)\right]}{\left[\pi\left(\frac{f}{R_{S}}-i\right)\right]^{2}} d f \\
\left.+C_{2} \int_{f_{c}-W_{b} / 2}^{f_{c}-W_{b} / 4} f \frac{\sin ^{2}\left[\pi\left(\frac{f}{R_{S}}-i\right)\right]}{\left[\pi\left(\frac{f}{R_{S}}-i\right)\right]^{2}} d f\right]
\end{array}\right]
\end{aligned}
$$

In (6), let $u=\pi\left(\frac{f}{R_{S}}-i\right), h_{1}=f_{C} / R_{S-} W b / 2 R_{S}$, and $h_{2}=f_{C} / R_{S}-W b / 4 R_{S}$. Then (6) can be rewritten as follows:

$$
\begin{aligned}
& \int_{f_{c}-W_{b} / 2}^{f_{c}-W_{b} / 4} A_{1}(f) d f=\sum_{i=0}^{M-1} C_{1} \frac{P_{S}}{\pi}\left[\frac{R_{S}}{\pi} \int_{\pi\left(h_{1}-i\right)}^{\pi\left(h_{2}-i\right)} \frac{\sin ^{2} u}{u} d u+i R_{S} \int_{\pi\left(h_{1}-i\right)}^{\pi\left(h_{2}-i\right)} \frac{\sin ^{2} u}{u^{2}} d u\right] \\
& +\sum_{i=0}^{M-1} C_{2} \frac{P_{S}}{\pi} \int_{\pi\left(h_{1}-i\right)}^{\pi\left(h_{2}-i\right)} \frac{\sin ^{2} u}{u^{2}} d u
\end{aligned}
$$

Using the trigonometric functions powers, $\sin 2 x=(1-\cos 2 x) / 2$, the integrals in (7) can be evaluated using [ref. 9] as follows,

$$
\begin{aligned}
& \int_{b}^{b} \frac{\sin ^{2} u}{u} d u=\frac{1}{2} \int_{a}^{b} \frac{1-\cos 2 u}{u} d u=\frac{1}{2}\left[\int_{a}^{b} \frac{1}{u} d u-\int_{a}^{b} \frac{\cos 2 u}{u} d u\right] \\
& \int_{b}^{b} \frac{\sin ^{2} u}{u^{2}} d u=\frac{1}{2} \int_{a}^{b} \frac{1-\cos 2 u}{u^{2}} d u=\frac{1}{2}\left[\int_{a}^{b} \frac{1}{u^{2}} d u-\int_{a}^{b} \frac{\cos 2 u}{u^{2}} d u\right]
\end{aligned}
$$


Fro $\mathrm{m}$ (8) and (9), $\int_{a}^{b} \frac{1}{u} d u=\ln b-\ln a$; and $\int_{a}^{b} \frac{1}{u^{2}} d u=\frac{1}{a}-\frac{1}{b}$

Also using MacLaurine series in [9]

$\int_{a}^{b} \frac{\cos 2 u}{u} d u=\ln b+\sum_{k=1}^{\infty}(-1)^{k} \frac{(2 b)^{2 k}}{2 k \cdot(2 k) !}-\left[\ln a+\sum_{k=1}^{\infty}(-1)^{k} \frac{(2 a)^{2 k}}{2 k \cdot(2 k) !}\right]$

$\int_{a}^{b} \frac{\cos 2 u}{u^{2}} d u=\frac{\cos 2 a}{a}-\frac{\cos 2 b}{b}-2 \int_{a}^{b} \frac{\sin 2 u}{u^{2}} d u$

$\int_{a}^{b} \frac{\sin 2 u}{u^{2}} d u=\sum_{k=1}^{\infty} \frac{(-1)^{k-1}}{(2 k-1)(2 k-1) !} \times\left[(2 b)^{2 k-1}-(2 a)^{2 k-1}\right]$

Therefore, employing (10), (11), and (12), both (8) and (9) can be written as (13) and (14), respectively,

$$
\begin{aligned}
& \int_{b}^{b} \frac{\sin ^{2} u}{u} d u=\frac{1}{2} \sum_{k=1}^{\infty}(-1)^{k} \frac{(2 a)^{2 k}-(2 b)^{2 k}}{2 k \cdot(2 k) !} \\
& \int_{b}^{b} \frac{\sin ^{2} u}{u^{2}} d u=\frac{1}{2}\left[\frac{1}{a}-\frac{1}{b}-\frac{\cos 2 a}{a}+\frac{\cos 2 b}{b}+2 \sum_{k=1}^{\infty} \frac{(-1)^{k-1}\left[(2 b)^{2 k-1}-(2 a)^{2 k-1}\right]}{(2 k-1)(2 k-1) !}\right]
\end{aligned}
$$

Employing (13) and (14), (7) can be represented as (15)

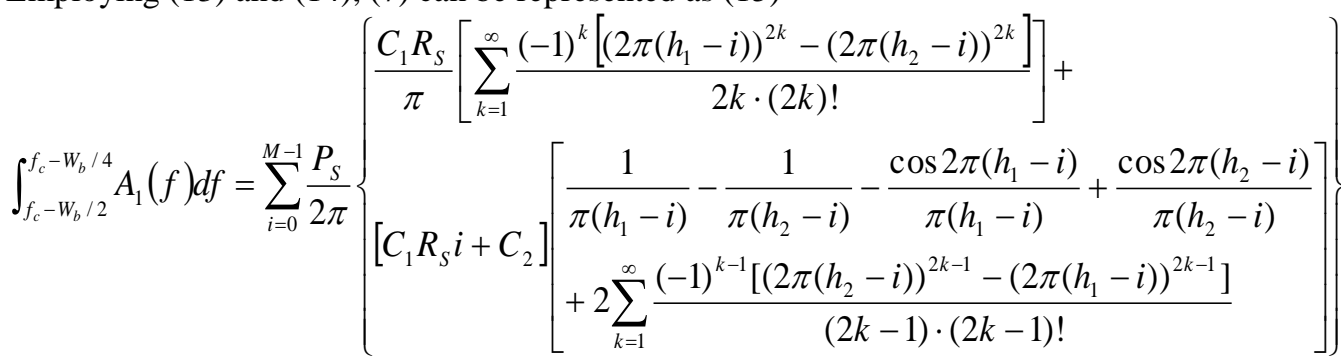

$$
\begin{aligned}
\int_{f_{c}-W_{b} / 4}^{f_{c}+W_{b} / 4} A_{2}(f) d f=\int_{f_{c}-W_{b} / 4}^{f_{c}+W_{b} / 4} S_{S}(f) d f & =\int_{f_{c}-W_{b} / 4}^{f_{c}+W_{b} / 4} \sum_{i=0}^{M-1} \frac{P_{S}}{R_{S}} \sin c^{2}\left(\frac{f}{R_{S}}-i\right) d f \\
& =\sum_{i=0}^{M-1} \frac{P_{S}}{R_{S}} \int_{f_{c}-W_{b} / 4}^{f_{c}+W_{b} / 4} \frac{\sin c^{2}\left[\pi\left(\frac{f}{R_{S}}-i\right)\right]}{\left[\pi\left(\frac{f}{R_{S}}-i\right)\right]} d f
\end{aligned}
$$

In (6), let $u=\pi\left(\frac{f}{R_{S}}-i\right), h_{2}=f_{C} / R_{S}-W b / 4 R_{S}$, and $h_{3}=f_{C} / R_{S}+W b / 4 R_{S}$. Then (16) can be rewritten as follows:

$$
\int_{f_{c}-W_{b} / 4}^{f_{c}+W_{b} / 4} A_{2}(f) d f=\sum_{i=0}^{M-1} \frac{P_{S}}{\pi} \int_{\pi\left(h_{2}-i\right)}^{\pi\left(h_{3}-i\right)} \frac{\sin ^{2} u}{u^{2}} d u
$$

The equation (14) can be used to evaluate (17), so

$$
\int_{f_{c}-W_{b} / 4}^{f_{c}+W_{b} / 4} A_{2}(f) d f=\sum_{i=0}^{M-1} \frac{P_{S}}{2 \pi}\left[\begin{array}{l}
\frac{1}{\pi\left(h_{2}-i\right)}-\frac{1}{\pi\left(h_{3}-i\right)}-\frac{\cos 2 \pi\left(h_{2}-i\right)}{\pi\left(h_{2}-i\right)}+\frac{\cos 2 \pi\left(h_{3}-i\right)}{\pi\left(h_{3}-i\right)} \\
+2 \sum_{k=1}^{\infty} \frac{(-1)^{k-1}\left[\left(2 \pi\left(h_{3}-i\right)\right)^{2 k-1}-\left(2 \pi\left(h_{2}-i\right)\right)^{2 k-1}\right]}{(2 k-1) \cdot(2 k-1) !}
\end{array}\right]
$$

By the same way it is easy to get 


$$
\begin{aligned}
& \int_{f_{c}+W_{b} / 4}^{f_{c}+W_{b} / 2} A_{3}(f) d f=\int_{f_{c}+W_{b} / 4}^{f_{c}+W_{b} / 2}\left(-C_{1} f+C_{3}+P_{S}\right) S_{S}(f) d f \\
& =\int_{f_{c}+W_{b} / 4}^{f_{c}+W_{b} / 2}\left(-C_{1} f+C_{3}+P_{S}\right) \sum_{i=0}^{M-1} \frac{P_{S}}{R_{S}} \sin c^{2}\left(\frac{f}{R_{S}}-i\right) d f \\
& =\sum_{i=0}^{M-1} \frac{P_{S}}{R_{S}}\left[\begin{array}{l}
-C_{1} \int_{f_{c}+W_{b} / 4}^{f_{c}+W_{b} / 2} f \frac{\sin ^{2}\left[\pi\left(\frac{f}{R_{S}}-i\right)\right]}{\left[\pi\left(\frac{f}{R_{S}}-i\right)\right]^{2}} d f \\
+\left[C_{3}+P_{S}\right] \int_{f_{c}+W_{b} / 4}^{f_{c}+W_{b} / 2} f \frac{\sin ^{2}\left[\pi\left(\frac{f}{R_{S}}-i\right)\right]}{\left[\pi\left(\frac{f}{R_{S}}-i\right)\right]^{2}} d f
\end{array}\right]
\end{aligned}
$$

In (19), let $u=\pi\left(\frac{f}{R_{S}}-i\right), h_{3}=f_{C} / R_{S}+W b / 4 R_{S}$, and $h_{4}=f_{C} / R_{S}+W b / 2 R_{S}$. Then by employing (13) and (14), (19) can be represented as (20)

$$
\int_{f_{c}-W_{b} / 2}^{f_{c}-W_{b} / 4} A_{3}(f) d f=\sum_{i=0}^{M-1} \frac{P_{S}}{2 \pi}\left\{\begin{array}{l}
\frac{-C_{1} R_{S}}{\pi}\left[\sum_{k=1}^{\infty} \frac{(-1)^{k}\left[\left(2 \pi\left(h_{3}-i\right)\right)^{2 k}-\left(2 \pi\left(h_{4}-i\right)\right)^{2 k}\right]}{2 k \cdot(2 k) !}\right]+ \\
{\left[\begin{array}{l}
\frac{1}{\pi\left(h_{3}-i\right)}-\frac{1}{\pi\left(h_{4}-i\right)}-\frac{\cos 2 \pi\left(h_{3}-i\right)}{\pi\left(h_{3}-i\right)} \\
+\frac{\cos 2 \pi\left(h_{4}-i\right)}{\pi\left(h_{4}-i\right)} \\
+2 \sum_{k=1}^{\infty} \frac{(-1)^{k-1}\left[\left(2 \pi\left(h_{4}-i\right)\right)^{2 k-1}-\left(2 \pi\left(h_{3}-i\right)\right)^{2 k-1}\right]}{(2 k-1) \cdot(2 k-1) !}
\end{array}\right]}
\end{array}\right\}
$$

By combine (15), (18), and (20), Therefore, Interfering signal power attenuation by bandwidth overlapping ratio can be expressed as:

$$
\left.L_{a t t}=10 \log _{10}\left\{\begin{array}{l}
\frac{C_{1} R_{S}}{\pi}\left[\sum_{k=1}^{\infty} \frac{(-1)^{k}\left[\left(2 \pi\left(h_{1}-i\right)\right)^{2 k}-\left(2 \pi\left(h_{2}-i\right)\right)^{2 k}\right]}{2 k \cdot(2 k) !}\right]+ \\
{\left[\begin{array}{l}
\frac{1}{\pi\left(h_{1}-i\right)}-\frac{1}{\pi\left(h_{2}-i\right)}-\frac{\cos 2 \pi\left(h_{1}-i\right)}{\pi\left(h_{1}-i\right)}+\frac{\cos 2 \pi\left(h_{2}-i\right)}{\pi\left(h_{2}-i\right)} \\
+2 \sum_{k=1}^{\infty} \frac{(-1)^{k-1}\left[\left(2 \pi\left(h_{2}-i\right)\right)^{2 k-1}-\left(2 \pi\left(h_{1}-i\right)\right)^{2 k-1}\right]}{(2 k-1) \cdot(2 k-1) !}
\end{array}\right]} \\
{\left[\begin{array}{l}
\left.\frac{1}{\pi\left(h_{2}-i\right)}-\frac{1}{\pi\left(h_{3}-i\right)}-\frac{\cos 2 \pi\left(h_{2}-i\right)}{\pi\left(h_{2}-i\right)}+\frac{\cos 2 \pi\left(h_{3}-i\right)}{\pi\left(h_{3}-i\right)}\right] \\
+2 \sum_{k=1}^{\infty} \frac{(-1)^{k-1}\left[\left(2 \pi\left(h_{3}-i\right)\right)^{2 k-1}-\left(2 \pi\left(h_{2}-i\right)\right)^{2 k-1}\right]}{(2 k-1) \cdot(2 k-1) !}
\end{array}\right]+} \\
{\left[\begin{array}{l}
-C_{1} R_{S}\left[\sum_{k=1}^{\infty} \frac{(-1)^{k}\left[\left(2 \pi\left(h_{3}-i\right)\right)^{2 k}-\left(2 \pi\left(h_{4}-i\right)\right)^{2 k}\right]}{2 k \cdot(2 k) !}\right] \\
{\left[\begin{array}{l}
\frac{1}{\pi}-\frac{1}{\pi\left(h_{4}-i\right)}-\frac{\cos 2 \pi\left(h_{3}-i\right)}{\pi\left(h_{3}-i\right)}+\frac{\cos 2 \pi\left(h_{4}-i\right)}{\pi\left(h_{4}-i\right)} \\
{\left[C_{3}-C_{1} R_{S} i+P_{S}\right]} \\
+2 \sum_{k=1}^{\infty} \frac{(-1)^{k-1}\left[\left(2 \pi\left(h_{4}-i\right)\right)^{2 k-1}-\left(2 \pi\left(h_{3}-i\right)\right)^{2 k-1}\right]}{(2 k-1) \cdot(2 k-1) !}
\end{array}\right.}
\end{array}\right]}
\end{array}\right]\right)
$$


Main contribution here that assuming the waveform of FSS signal is tringular, which is represents the real case of interfered scenario.

\section{Simulation Results and Discussion}

The IMT system occupies a bandwidth of $40 \mathrm{MHz}$ assigned with a centre frequency of $3500 \mathrm{MHz}$. A dish-shaped parabolic antenna, having a diameter of $2.4 \mathrm{~m}$ and a maximum antenna gain of $42.5 \mathrm{dBi}$, is deployed. The permissible long-term interference power is considered to be $-166 \mathrm{dBW} / 40 \mathrm{MHz}$, which is calculated based on $\mathrm{I}=\mathrm{N}-10 \mathrm{~dB}$ for $20 \%$ of the time. Currently, the specifications for the IMT-Advanced systems are under consideration. We assume a cellular OFDM/OFDMA of IMT-Advanced systems. The other parameters for the base station (BS) of IMT-Advanced systems are assumed as time division duplex (TDD), $10.24 \mathrm{kHz}$ subcarrier frequency spacing, $80 \mathrm{MHz}$ channel bandwidth, 8192 subcarriers, and $13 \mathrm{dBW}$ transmit power. The BS antenna pattern used for each sector is specified as $25 \mathrm{~dB}$ maximum antenna gain

Based on the system parameters of FSS and IMT-Advanced systems, the minimum separation distance to satisfy permissible interference power is analyzed and compared for both A-MCL and BIM due to the BS transmit power of the IMT-Advanced system. Here cluster loss is assumed as $20 \mathrm{~dB}$. For antenna discrimination loss the cases of $50 \mathrm{~dB}$ are considered. Figure 2 depicts the required minimum separation distance against BS transmit power of an IMT-Advanced system for both a A-MCL and BIM. The BIM results show more stable values than the A-MCL at the same interference power on the victim system.

The maximum difference of the interfering signal power between A-MCL and BIM is $3 \mathrm{~dB}$ and the maximum difference of the required minimum separation distance between conventional MCL and A-MCL is approximately $12 \mathrm{~km}$. The BIM method reflects the capability of flexible spectrum usage for OFDM-based IMT-Advanced systems and decreases the received.

References

[1]. "A comparison of the minimum coupling loss method, enhanced minimum coupling loss method, and the monte-carlo simulation," CEPT ERC Report 101, May 1999.

[2]. W.-G. Chung, H.-S. Jo, H.-G. Yoon, J.-W. Lim, J.-G. Yook and H.-K. Park. "Advanced MCL method for sharing analysis of IMTadvanced systems", ELECTRONICS LETTERS 12th October 2006 Vol. 42 No. 21.

[3]. CEPT ECC Report 68, Compatibility Studies In The Band 5725-5875MHz Between Fixed Wireless Access (FWA) Systems And Other Systems, 2005.

[4]. "Adjacent band compatibility between UMTS and other services in the 2GHz band," CEPT ERC Report 65, May 1999.

[5]. Han-Shin Jo, Hyun-Goo Yoon, JaeWoo Lim and Jong-Gwan Yook "An Advanced MCL Method for Assessing Interference Potential of OFDM-Based Systems beyond 3G with Dynamic Power Allocation" Proceedings of the 9th European Conference on Wireless Technology, UK 2006.

[6]. ITU-R Recommendation F. 758-3: 'Considerations in the development of criteria for sharing between the terrestrial fixed service and other services' 